\title{
Bereitschaftspotential augmentation by neuro-feedback training in Parkinson's disease
}

\section{$\operatorname{AUTHOR}(\mathrm{S})$ :}

Fumuro, Tomoyuki; Matsuhashi, Masao; Mitsueda, Takahiro; Inouchi, Morito; Hitomi, Takefumi; Nakagawa, Tomokazu; Matsumoto, Riki; ... Mima, Tatsuya; Takahashi, Ryosuke; Ikeda, Akio

\section{CITATION:}

Fumuro, Tomoyuki ...[et al]. Bereitschaftspotential augmentation by neuro-feedback training in Parkinson's disease. Clinical Neurophysiology 2013, 124(7): 1398-1405

\section{ISSUE DATE:}

2013-07

URL:

http://hdl.handle.net/2433/175537

\section{RIGHT:}

(c) 2013 International Federation of Clinical Neurophysiology. Published by Elsevier Ireland Ltd.; この論文は出版社版でありません。引用の際には出版社版をご確認ご利用 ください。; This is not the published version. Please cite only the published version. 


\section{[Title]}

Bereitschaftspotential augmentation by neuro-feedback training in Parkinson's disease

\section{[Authors]}

Tomoyuki Fumuro ${ }^{1}$, Masao Matsuhashi ${ }^{2}$, Takahiro Mitsueda ${ }^{1}$, Morito Inouchi ${ }^{1}$, Takefumi

Hitomi ${ }^{1}$, Tomokazu Nakagawa ${ }^{1}$, Riki Matsumoto ${ }^{1}$, Jun Kawamata ${ }^{1}$, Haruhisa Inoue ${ }^{1}$, Tatsuya

Mima $^{2}$, Ryosuke Takahashi ${ }^{1}$, Akio Ikeda ${ }^{1}$

${ }^{1}$ Department of Neurology, Kyoto University Graduate School of Medicine

${ }^{2}$ Human Brain Research Center, Kyoto University Graduate School of Medicine

[Address for correspondence and proofs]

[Name]

Akio Ikeda, MD, $\mathrm{PhD}$

[Address]

Department of Neurology, Kyoto University Hospital

54 Kawaharacho, Shogoin, Sakyo-ku, Kyoto, 606-8507, Japan

[Telephone and fax number] 
Tel: (+81)-75-751-3772, Fax: (+81)-75-751-9416

\section{[E-mail address]}

akio@kuhp.kyoto-u.ac.jp

\section{[Key Words]}

neuro-feedback (NFB), slow cortical potentials (SCPs), Bereitschaftspotential (BP), Parkinson's

disease (PD), supplementary motor area (SMA)

Number of references

Number of figures

Number of tables

Title characters

Abstract words

Acknowledgement

Text words

Key words
32

8

2

$78 / 135$

$200 / 200$

1

6186 (include reference, not limited)

$5 / 6$ 


\section{Acknowledgements}

No conflict of interests is present by the authors.

This study was supported by the Research Grant (22A-6) for Nervous and Mental Disorders from the Ministry of Health and Welfare, the Grants-in-Aid for Scientific Research (23591275) from the Japan Society for the Promotion of Science (JSPS), the Research Grant from the Japan Epilepsy Research Foundation (2009-2011) and the Strategic Research Program for Brain Sciences (SRPBS) from the MEXT of Japan.

International Federation of Clinical Neurophysiology (IFCN) presented this study with the fellowship award at 29th Internal Congress of Clinical Neurophysiology (ICCN2010). 


\section{Highlights}

- This study showed that PD patients could restore decreased early component of BP by means of the neuro-feedback (NFB) to control negative slow cortical potentials (SCPs).

- It was the first study to demonstrate that decreased pre-movement cortical activity can be restored by endogenous, subject's own effort, without externally driven modulatory stimuli or medication.

- Good NFB performance of negative SCPs shifts (negativation) most likely increases excitatory field potentials of pyramidal cells in the supplementary motor area. 


\section{Abstract}

Objective: Decreased early Bereitschaftspotential (BP) is one of the electrophysiological characteristics in patients with Parkinson's disease (PD). We examined whether PD patients could increase BP amplitude by means of neuro-feedback (NFB) training for their slow cortical potentials (SCPs).

Methods: We employed 10 PD patients and 11 age-matched controls. BP was measured for self-paced button-pressing by their right thumb. Subjects were instructed to make the introspective efforts to produce negative SCPs (negativation). One-day session consisted of 3 trials, i.e., the first BP, NFB training and the second BP, and each patients performed for $2 \sim 4$ days. Amplitudes of the first and second BPs were compared between the 2 groups that were divided depending on NFB performance.

Results: Good NFB performance had the tendency of larger early BP in the second BP recording than in the first one, whereas in the poor NFB performance the early BP was smaller in the second BP recording than in the first one in both patient and normal groups $(p<0.001)$.

Conclusions: Good NFB performance for negativation could increase excitatory field potentials of pyramidal cells for the generation of early BP.

Significance: Voluntary regulation of SCPs could enhance BP in PD patients and in aged controls. 


\section{Introduction}

Parkinson's disease (PD) is a degenerative disorder of the dopaminergic neurons of the central nervous system that impairs the motor skills, speech and other functions (Jankovic, 2008). It is characterized by muscle rigidity, tremor, a slowing of physical movements (bradykinesia) and even a loss of physical movements (akinesia) in the extreme situation. The primary motor symptoms are the results of decreased activities of the motor cortices through the basal ganglia-thalamo-cortical loop, caused by the insufficient formation and action of dopamine, which is produced in the dopaminergic neurons of the brain (Zaidel et al., 2009). Anatomical studies of the primate basal ganglia systems show that a major portion of pallidal output is directed to the non-primary motor areas of frontal cortex, in particular to the supplementary motor area (SMA) (Jurgens, 1984; Schell and Strick, 1984). In human, it was reported that the SMA is crucial to the organization of both initial as well as sequential movements (Roland et al., 1980; Roland et al., 1982) and an abnormality of sequencing hand and elbow movements has been demonstrated in patients with unilateral SMA damage (Dick et al., 1986). A similar disturbance of sequenced hand and elbow movements has been demonstrated in patients with PD (Benecke et al., 1987), suggesting impairment of SMA function.

Bereitschaftspotential (BP) is a negative slow electroencephalographic (EEG) potential preceding self-paced movement (Deecke and Kornhuber, 1978). BP consists of an early- and late 
components (Tamas and Shibasaki, 1985; Shibasaki and Hallett, 2006). The early component of BP could reflect the activity of SMA as its maximum amplitude at the vertex (overlying SMA) regardless of the body parts involved in the movements (i.e., eye, hand, arm or foot) (Deecke Kornhuber, 1978). The late component is lateralized to the hemisphere contralateral to the side movements and is considered to represent activity mainly of the primary motor cortices (Shibasaki et al., 1978; Ikeda et al., 1992).

Dick et al. reported that the early component of BP was smaller in PD patients than in control subjects whereas its late component was not (Dick et al., 1989). Furthermore, the amplitude of early component of BP was shown to be smaller in the off-phase of L-DOPA medication and returned to normal in the on-phase (Dick et al., 1987).

Scalp-recorded EEG is comprised of very wide range of oscillatory activities from delta to gamma activities, and of even direct current (DC) potential shifts, that we call as slow cortical potentials (SCPs) in this report. Negative and positive SCP shifts reportedly reflect increase and decrease of the excitability of underlying cortical networks (Birbaumer et al., 1990). In epilepsy patients, negative SCPs are recorded at the time of seizure onset as ictal EEG changes (Ikeda et al., 1996). In recent years, the neuro-feedback (NFB) training was launched as an attempt to reduce seizure frequency by regulating their SCPs presumably as positive as possible in polarity (positivation) (Kotchoubey et al., 1999). In their study Cz electrode was 
used to record and control SCP S and the authors showed that cortical excitability could be modified through the effort to control one's own SCPs. Using the NFB system, once they were well trained and became capable of modifying SCPs between the negativity and the positivity shifts, the degree of seizure control became better in well trained group than poorly trained group (Kotchoubey et al., 2001). We previously reported that, after enough training to regulate their SCPs by means of NFB, young normal subjects produced a larger early component of BP while they were trying to produce negative SCPs shifts (negativation), as compared with BP obtained before NFB training (Fumuro et al., 2010). In contrast, no significant differences were observed in the late component of BP between the 2 conditions. These results suggested that the self-regulated negative SCPs (negativation) may activate the generators of early component of BP.

The aim of this study was to clarify whether PD patients could restore their BP amplitude by means of NFB to control negative SCPs (negativation). We therefore, investigated the effects of self-regulation of SCPs on the BP amplitude from 10 PD patients and from 11 age-matched normal controls. 


\section{Methods}

\subsection{Subjects}

We investigated 10 right-handed patients ( 2 men and 8 women) clinically diagnosed as PD (10 patients) aged 36-71 (average of 63) years. Their clinical features are summarized in Table 1. 11 right-handed normal controls (1 man and 10 women) aged 60-69 (average of 65) years were also investigated as controls. Dominant hand was assessed by the Edinburgh Handedness Inventory. In patients with PD, this study was done while medication was on (Table 1). All subjects made the written informed consent about the study. This study was approved by the Ethics Committee of Kyoto University School of Medicine (No. E-308).

\subsection{Recording condition}

$5 \mathrm{Ag} / \mathrm{AgCl}$ scalp electrodes placed at $\mathrm{C} 3, \mathrm{C} 1, \mathrm{Cz}, \mathrm{C} 2$ and $\mathrm{C} 4$ according to the International 10-10 System were used for EEG recording. Electrooculogram (EOG) was recorded for horizontal and vertical eye movements by placing the 4 skin electrodes on the bilateral outer canthi, and on the upper and lower edges of the left orbital, respectively. Electromyogram (EMG) was recorded from the right abductor pollicis brevis muscle by a pair of skin electrode over the muscle. 
EEG, EOG, EMG and input signal by pressing the button with the subject's right hand were recorded using a DC-EEG feedback system NEURO PRAX (neuroConn GmbH, Ilmenau, Germany) (Schellhorn et al., 2004).

Since the main concern of this study was to record SCPs or DC potentials reliably, we confirmed the following 4 conditions completely (Fumuro et al., 2010).

1) NEURO PRAX contains a DC differential amplifier, which has a huge size of input impedance of $>10$ gigaohm. The huge size of input impedance minimizes the effect of electrode impedance and further enables us to record stable DC potentials without a high-pass filter (Cooper et al., 1980).

2) Sintered $\mathrm{Ag} / \mathrm{AgCl}$ electrodes were soaked into Ten20 conductive paste (Weaver and Company, Aurora, USA) for $>30$ min before measurement in order to stabilize polarization potential of electrodes. These electrodes had lower polarization potentials and were shown to provide stable recording condition (Tallgren et al., 2005).

3) Before electrode attachment, the skin to place the surface electrodes was cleaned by both cleansing paste (Skin Pure; Nihon Kohden, Tokyo, Japan) and sanitary cotton moistened with ethanol, and then electrodes as described above were firmly attached.

4) Before actual recording sessions, we checked and confirmed that the offset voltages of all the electrodes were within $\pm 20 \mathrm{mV}$ range relative to the $\mathrm{A} 1$, and the standard deviation of the drifts 
were $<1000 \mu \mathrm{V}$. We also visually confirmed that there was no noticeable, even very low amplitude or very slow artifacts on the EEG waveforms before and throughout the measurement. Ordinary impedance check with $10 \mathrm{~Hz}$ alternating current used for conventional EEG, i.e., AC potential, recording was not performed.

All signals were digitized at the sampling rate of $128 \mathrm{~Hz}$ and band-pass filter from DC to $30 \mathrm{~Hz}$ was applied. The subjects sat in a chair comfortably with their arms and hands on the armrests. A computer screen was placed in front of the subjects by $130 \sim 150 \mathrm{~cm}$ from their faces. Eye movement correction program built in the NEURO PRAX was used to diminish eye movement artifacts. EEG signals were referred to A2 during both NFB training and BP recording.

\subsection{Task}

\subsubsection{Procedural overview}

Subjects performed 3 kinds of tasks in one day in the following order: 1) the first BP recording, followed by 5 min of break, 2) NFB training to regulate SCPs (2-5 times of sub-sessions with 3 min of break), followed by 5 min of break, 3) the second BP recording while subjects made introspective efforts that produced the negative SCP shifts (negativation)

(Fig. 1). The subjects repeated the set of 1)-3) procedures (one-day session) as described above 2 to 4 times each in the different day, with 1-6 days of interval for each other. The BP recording 
and NFB training in the first day of each subject were regarded as the rehearsal sessions and thus those were not included for the final analysis. It was not informed to the subjects before examination.

\subsubsection{BP recording}

Subjects were instructed to look at the blank screen in front of them but not to fixate on a certain point. They were told not to blink from $3 \mathrm{sec}$ before to $1 \mathrm{sec}$ after the button-press. They performed self-paced, brisk button-press by their right thumb about every $10 \mathrm{sec}$. All subjects had approximately 100 trials of button-press, which was sufficient to obtain approximately 80 epochs of artifact-free trials. EEG signals were referred to A2 during BP recording, and the reference was changed to linked reference of $\mathrm{A} 1$ and $\mathrm{A} 2$ for $\mathrm{BP}$ analysis. $4 \mathrm{sec}$ of analysis epochs from -3 to $+1 \mathrm{sec}$ to the onset of button-press were cut out from the raw data in an off-line manner, EEG data during BP recording was first linearly detrended for each $4 \mathrm{sec}$ of EEG segment. Applied detrend could work for a linear fit (in the least-squares sense) to EEG data and then removes inappropriately occurring trend from it. For the epochs containing artifacts exceeding $150 \mu \mathrm{V}$ in amplitude (from negative peak to positive peak or vise versa) in the EEG channels were excluded. Artifact-free EEG epochs were precisely aligned to the trigger signal of button-press and averaged. EEG baseline was defined 
as the mean amplitude of the first $0.4 \mathrm{sec}$ of the epoch.

At least 50 artifact-free epochs were averaged. To confirm the reproducibility, the epochs were divided into 2 groups (odd and even numbers of epochs during BP recording), and were averaged to make 2 subensembles of waveforms. The sessions where 2 subensembles of BP were not reproducible were excluded from the further analysis.

We evaluated the following 3 measures (Fig. 2). 1) Early BP (E-BP): mean amplitude of the movement-related cortical potentials (MRCPs) between 0.6 and $0.4 \mathrm{sec}$ before the trigger onset. It corresponds to the early BP (Shibasaki et al., 1980; Shibasaki and Hallett, 2006).

2) Pre-movement negative peak (N1): the largest pre-movement negative potentials occurring between $-0.5 \mathrm{sec}$ and the trigger onset measured from the baseline. Since this study employed the button-press for the trigger onset instead of surface EMG onset, the pre-movement potentials often peaked immediately before the button-press trigger onset, and thus its amplitude could contain the whole BP and motor potential in Shibasaki and Hallett (2006). Therefore, we termed it as pre-movement negative peak.

3) Late-BP (L-BP): this steeper negativity was obtained by subtracting the E-BP from N1, and arbitrarily referred to the L-BP. It maintly corresponds to NS') (Shibasaki et al., 1980; Shibasaki and Hallett, 2006) although it may partly contain motor potential as explained just above. 


\subsubsection{NFB: self-regulation of SCPs}

NFB training to regulate SCPs was done after 5 min of break following the first BP

recording. The procedure was the same as the previous study (Kotchoubey et al., 2001; Fumuro

et al., 2010).

SCPs recorded from $\mathrm{Cz}$ were continuously shown on the front screen as a slowly

moving sunfish from the left to the right by $8 \mathrm{sec}$ as an almost real time demonstration (the

feedback time delay was around $150 \mathrm{msec}$ ) (Fig. 3). It was also shifting upward (negative shift or negativation) or downward (positive shift or positivation), according to the change of SCPs.

Namely, the sunfish worked as a visual feedback of the subjects' own SCPs at Cz. Once the sunfish appeared at the left edge, the dichotic instruction symbols appeared in the center of the screen: the upward or downward arrow-head at the same time (Fig. 3). The direction of the apex of the arrow-head, i.e., up or down, indicated the polarity to which the subjects should shift their SCPs.

The subjects were told to regulate the SCPs upon the instruction symbols, and by means of this real-time feedback of SCPs at Cz the subjects tried to learn how to control it by themselves. Therefore, any specific suggestions how to regulate SCPs were not initially provided by the examiners; subjects were advised to concentrate in the SCP shift and to find the most successful strategy to move the sunfish toward the required direction. The examiner gave 
them some examples of mental introspection to control SCPs which were done and useful in the young subjects in our previous study (Fumuro et al., 2010), if they wanted to know.

Each trial lasted $10 \mathrm{sec}$, which was from $2 \mathrm{sec}$ before to $8 \mathrm{sec}$ after appearing the instruction symbols on the center of the screen. Next trial was separated from the previous trial by random intervals of 1 to $6 \mathrm{sec}$. Negative- and positive feedback trials were presented pseudo-randomly at equal probability. NFB training sub-sessions was conducted 2 to 5 times in one day, and each sub-session had 52 trials. The number of session was determined based on each subject's condition and fatigability.

Since this training procedure was expected to be done finally without the apparatus, trials with and without feedback information (the moving sunfish) were intermixed pseudo-randomly with the equal number, the latter called as transfer trials (Fig. 3 (A) and (B): lower half).

The trial was judged as successful for feedback control once the SCP amplitude exceeded a defined target level and remained at least for $2 \mathrm{sec}$ in the last $4 \mathrm{sec}$ of the trial. This target level was set by the examiner as follows: in the negative shift trials, the level was set to the range from the baseline to -30 to $-50 \mu \mathrm{V}$, and in the positive shift trials it was from the baseline to +30 to $+50 \mu \mathrm{V}$. The EEG baseline was obtained from the first $1 \mathrm{sec}$ of the trial. In case of successful trial, another graphic symbol ('the shining sun') appeared as a reward mark 
(Fig. 3 (A): right). The reward marks were also presented in transfer trials when judged as successful. The data in the transfer trials were employed for the judgment of performance as described later in the method. The effective visual field for each eye was described below; sunfish: $3 \times 3$ (degree in horizontal $x$ vertical), arrow-head: $1-2 \times 3$, the shining sun: $6-8 \times 5-7$, width of display: 9-10x11-13.

After each sub-session, the experimenter asked the subjects their introspective strategies and encouraged them to renew strategies if the result of the previous sub-session did not have many successful trials. 


\section{Analysis}

\subsection{Analysis of NFB (Table 2. upper half)}

EEGs of the transfer trials in the latter half of the 2-5 sub-sessions out of one-day

training were processed by high frequency filter of $2 \mathrm{~Hz}$, and then separately averaged for the negativity and positivity trials. (In case of a total of 3 or 5 sub-sessions, the $2^{\text {nd }}$ or $3^{\text {rd }}$ sub-session was included in the average). Trials containing artifacts exceeding $200 \mu \mathrm{V}$ (peak to peak) in any of the EEG channels were excluded from average.

A one-day session was classified as a good NFB performance if the averaged waveforms of the negativity trials exceed that of the positivity trials at least 3 electrodes including $\mathrm{Cz}$ in the period of 2 to $6 \mathrm{sec}$ after the visual symbol appeared on the left (Fig. 4: left). Otherwise, it was classified as a poor NFB performance (Fig. 4: right).

\subsection{Analysis of BP (Table 2: lower half)}

All statistical analyses were performed with SPSS (version 15.0, SPSS Inc., Chicago, Illinois, USA). Analysis was performed for $3 \mathrm{BP}$ components (E-BP, N1 and L-BP) at Cz because that means difference between the first and second BP amplitudes were the most prominent at $\mathrm{Cz}$ as compared with other electrodes, and that the visual feedback of the subjects' own SCPs was recorded from $\mathrm{Cz}$ during NFB training. The Shapiro-Wilk test was used to 
assess for normality; if normality is accepted, analysis of variance (ANOVA) was used.

Otherwise, the Mann-Whitney U test was used. In order to take the multiplicity of comparisons into account, $p$ values were corrected with Holm's correction in all statistical analyses. In order to strengthen the findings, we have analyzed all the data in the session-based and subject-based analyses as follows.

\subsubsection{Session-based analysis (Figs. 5, 6 and 7)}

The first one-day sessions in all the subjects, and any one-day sessions without reproducibility of subensembles of $\mathrm{BP}$ waveform were excluded, and then mean difference between the first and second BP amplitudes was compared. In subjects who had both good- and poor NFB performance, those one-day sessions were separately enrolled in both good- and poor NFB performance, respectively. Two-way ANOVA having 2 between-subjects factor (1. disease: PD patients and normal controls, 2. NFB performance: good and poor NFB performance) was conducted to analyze mean difference between the first and the second BP amplitudes at $\mathrm{Cz}$.

\subsubsection{Subject-based analysis}

Similar to the session-based analysis, the first one-day sessions in all the subjects, and any one-day sessions without reproducibility of subensembles of BP waveform were excluded, 
and then mean difference between the first and second BP amplitudes was compared in each

subject. The last one-day session was adopted for the analysis of mean difference between the

first and second BP amplitudes. All subjects were finally divided into two-subject analysis

groups. Two-way ANOVA was conducted to analyze mean difference between the first and the second BP amplitudes in the same manner as the session-based analysis.

\subsubsection{Comparison of BP between PD patients and normal controls}

A statistical analysis with Mann-Whitney U test was performed to assess the difference of the first and second $\mathrm{BP}$ between $\mathrm{PD}$ patients and normal controls at $\mathrm{Cz}$ regardless the degree of their NFB performance. It adopted the data of the session-based BP analysis. 


\section{Results}

\subsection{Performance of NFB （Table 2: upper half）}

In PD patients, out of a total of 27 one-day sessions, the first one-day sessions $(n=10)$

in 10 patients were automatically excluded from further analysis (It was because that in the

initial training period subjects could not be familiar with or not good at this internal

neuro-feedback process which could distort the finding erroneously). 8 one-day sessions were

grouped into good NFB performance and 9 sessions were into poor one. In normal controls, out

of a total of 31 one-day sessions, the first one-day sessions $(n=11)$ in 11 subjects were excluded automatically from further analysis. 12 one-day sessions were grouped into good NFB

performance and 8 one-day sessions were into poor one. To compare the degree of good

performance rate between the first one-day sessions and $2^{\text {nd }}-4^{\text {th }}$ one-day sessions, 10 and 11

one-day sessions in the first day was also judged for performance in PD and normals,

respectively. Good performance outcome rate was higher in the $2^{\text {nd }}-4^{\text {th }}$ one-day sessions than in the first one-day sessions (30\% vs. $47 \%$ in PD, $36 \%$ vs. $60 \%$ in normals) (although it did not reach the statistical significance).

The number of finally accepted one-day sessions of NFB were similar between the both patient and normal groups (17 vs. 20 ). The number of one-day session with good NFB performance was equally $47 \%$ and $60 \%$ in the both groups, respectively. 


\subsection{Effects of NFB on BP}

\subsubsection{Session-based analysis}

E-BP (Fig. 5, Fig. 6): Two-way ANOVA revealed significant main effect of NFB performance $(F(1,26)=21.077, p<0.001)$. No main effect of disease or interaction attained significance.

This means that regardless of PD or normal control, the second E-BP tended to be larger than the first one in the good NFB one-day sessions, whereas in the poor NFB performance the second E-BP tended to be smaller than the first one.

N1 (Fig. 5): Two-way ANOVA revealed significant main effect of NFB performance $(F(1,26)$

$=18.389, p<0.001)$. No main effect of disease or interaction attained significance. The result of N1 turned out to be the similar tendency as that of E-BP.

L-BP (Fig. 5): Two-way ANOVA revealed no significant main effect or interaction.

\subsubsection{Subject-based analysis (Table 2: lower half)}

As the subject-based analysis, 4 PD patients were grouped into good NFB group, and 3 patients were into poor one. In normal controls, 5 subjects were into good group, and 4 were into poor one.

Two-way ANOVA revealed significant main effect of NFB performance on E-BP ( $F(1$, 
$15)=12.607, p=0.006)$ and $\mathrm{N} 1(F(1,15)=18.800, p=0.002)$. No main effect of disease or interaction attained significance. Similar to session-based analysis, this means that regardless of PD or normal control, the second E-BP and N1 tended to be larger than the first ones in good NFB group, whereas the poor NFB group tended to have smaller E-BP and N1 in the second BP than in the first one. In contrast, no significant differences were observed in the L-BP (no figures shown).

\subsection{Comparison of BP between PD patients and normal controls (Fig. 8)}

Mann-Whitney U test revealed the significant difference of the session-based first BP between PD patients and normal controls in N1 $(p=0.035)$ and E-BP $(p=0.039)$. No significant difference was found in L-BP. It means that the first E-BP and N1 had smaller in PD than that in normal control, whereas L-BP did not reach the significant difference. The result of the second N1 and E-BP turned out to be the similar significant difference as the first one (N1: $p$ $=0.021, \mathrm{E}-\mathrm{BP}: p=0.039, \mathrm{~L}-\mathrm{BP}:$ not significant). 


\section{Discussion}

Brain plasticity, brain computer-interface and neuromodulation are the most important concerns in the current clinical neuroscience. Deep brain stimulation in patients with PD provided the great success at least to lessen the patients' symptoms. It belongs to the externally regulated, or exogenous procedure of neuromodulation. BP was modulated by TMS previously (Rossi et al., 2000), however, little has been investigated for intrinsic, endogenous procedure for BP modulation.

We previously reported that, after enough training to regulate their SCPs by means of NFB, young normal controls produced a larger early component of BP while they were trying to produce negative SCPs shifts (negativation), as compared with BP obtained before NFB training (Fumuro et al., 2010). In contrast, no significant differences were observed in the late component of BP between the 2 conditions. These results suggested that the self-regulated negative SCPs (negativation) could activate the generators of early component of BP.

The aim of this study was to clarify whether PD patients could restore their BP amplitude by means of NFB to control negative SCPs (negativation). This study presented the first scientific evidence that MRCPs could be restored by subject's own effort, instead of the external stimuli or medication in patients with PD and aged normal controls. As the results, with regard to the BP, similar to the previous study (Dick et al., 1989), PD patients had smaller early 
BP than aged normal controls, but not for late component of BP (Fig. 8). It suggests that the

situation in the present study of MRCP is very consistent with the previous study.

We could summarize the main finding as follows. Good NFB performance had the tendency of larger early BP in the second BP than in the first one, whereas the early BP tended to be smaller in the second BP than in the first one in the poor NFB performance in both PD and normal control (Figs. 5, 6 and 7).

The present study provides us with the very important concerns as follows. (1) Decreased BP in patients with PD could be potentially reversible as long as NFB was well performed or non-pharmacological process optimally works in the brain, and (2) neurophysiological BP modulation is mainly for its early component rather than the late one immediately before voluntary movement onset. With regard to (1), it was previously reported that administration of L-DOPA in normal controls could significantly increase the amplitude of BP (Dick et al., 1987). Namely, not only administration of dopaminergic drugs but also neurophysiological intrinsic activity commonly modify the brain activity. Deep brain stimulation could lessen the clinical symptoms of patients with PD, but it remains to be solved whether the present study or neuromodulation of BP would lessen the symptoms of patients with PD or not in the future study. As regards (2) or generators of BP, surface-negative BP is assumed to reflect field potential, probably excitatory postsynaptic potentials (EPSPs) in 
the superficial layer of the apical dendrite of cortical pyramidal neurons as the result of the thalamocortical input (Hashimoto et al., 1979). The current consensus of the generators of early component of BP is that it begins in the pre-SMA with no site-specificity and is followed by the SMA proper according to the somatotopic organization, as well as in the lateral motor cortices bilaterally again with relatively clear somatotopy (Ikeda et al., 1992; Ikeda et al., 1995; Yazawa et al., 2000).

Late component of $\mathrm{BP}$ occurs in the contralateral M1 and the lateral motor cortices with precise somatotopy (Neshige et al., 1988; Shibasaki and Hallett, 2006). With regard to the effect of NFB on the early BP component, the contribution of electrical fields arising from at least bilateral pre-SMA and SMA proper for the midline component. From this point of view, SMA activation by means of the self-regulated negative SCPs would act to restore E-BP in PD patients which showed hypofunction in the SMA as reported before. This implication is consistent with previous study by using the functional magnetic resonance imaging (fMRI): a strong positive correlation between negativation of SCP and blood oxygenation level-dependent (BOLD) contrasts was found in the hippocampus and the SMA (Hinterberger et al., 2003). According to these findings, the increased amplitude of early BP while negativation task was performed can be interpreted as efficient thalamo-cortical modulation via at least SMA. 
With regard to the cortical generators of scalp-recorded SCP, NFB in the present study may share the features of pursuit like visuo-motor performance using BP-trajectory definitely. 1) It could affect SCP amplitude because of co-occurrence of pursuit related BP. In addition, NFB in the present study also may share the features of the methods of contingent negative variation $(\mathrm{CNV})$, and at least the SMA is activated during CNV paradigm. During NFB performance, similar to the trials with visual feedback ('sunfish', in Fig. 3), subjects might have had an image of moving fish in their mind even during the trials without visual feedback. 2) It could affect the amplitude of second BP because of co-occurrence of memory-guided pursuit imaging CNV. For 1), the amplitude of scalp-recorded BP is usually rather small (several $\mu \mathrm{V}$ ) as compared with SCP amplitude (10-20 $\mu \mathrm{V})$, its effect could be practically little. For 2), it is still possible and should be carefully taken into account.

There is a different finding in BP between the present study and our previous one (Fumuro et al., 2010) in the poor performance situation as follows. In the previously study, young subjects showed increased early BP amplitude with good NFB performance in the second BP recording as compared with the first recording. In the poor NFB performance, they did not show significant difference of early BP between the first and second BP recording. In the present study, good NFB performance showed more or less the similar tendency of early $\mathrm{BP}$ as seen in the young normal controls, However, the early BP tended to be smaller in the 
second BP than that in the first one in the poor NFB performance in both PD and normal control (Figs. 5, 6 and 7). This tendency may reflect the effects of probably central fatigue in aged normal controls. In the present study, an entire examination of a session took 2 to 3 hours, including the time for preparation. The repetitive process of the button-press would cause the fatigue and result in the decrement of the BP usually (Dirnberger et al., 2004). In the good NFB performance, this decrement may be cancelled out or rather more exceed its former state by the good negative SCPs performance. In contrast, fatigue may directly affect on BP in the poor NFB performance. However, in the young subjects, the degree of fatigue would be just so small that the degree of NFB performance more directly affected on the BP amplitude. Another possible cause for this tendency is the difference level of adaptation for this study. As described in previous literature (Shibasaki and Hallett, 2006), more intention would result in larger amplitude of BP. For instance, in sessions with poor NFB performance, the subjects may have higher motor commands to execute the task in recording of first BP, then they may gradually adapt to the optimized commands or efforts to adjust the task, resulting in the normal motor control. Although sessions with poor adaptability were excluded from the final analysis, we could not completely exclude this possibility.

There are several limitations in the present study. Firstly, the terms of the NFB training were limited only 2 to 4 days. Because of short term of NFB training, we could not verify 
whether the each subject make NFB performance better day by day or not. Therefore, the grouping by one-day sessions required further validation as to whether the NFB performance improved much more with lesser artifact. In order to contain artifact-free data, as described previously (in the Methods), 1) the notion that averaged waveform of the SCPs should show a reproducibility was verified in several electrodes, 2) in BP measurement, epochs containing artifacts were excluded the in the objective manner, and 3) epochs were separately averaged into two BP waveforms, and any measurements with poorly-reproducible BP were excluded from further analysis. Through these processes, most artifacts should be excluded from the result and final analysis had the minimized artifact-effects. In addition, we compared the two BP waveforms that are measured in the same day, for reducing the effect of various factors such as level of intention and preparatory state that affect the magnitude of BP (Shibasaki and Hallett, 2006). Secondly, we put together all the PD patients with mild to severe degree of Hoehn and Yahr grade (H-Y grade) (Hoehn and Yahr, 1967) into one group. It is possible that each grade has different neurobiological mechanisms and therefore different BP characteristics. Thirdly, the underlying working mechanism of NFB training remains to be solved. It is probably closely associated with the guided activation theory, rather than reinforcement learning theory although there is some overlap between the two. It is also related to the free will but partly to the automatically guided action since the dichotic instruction symbols let subjects decide either 
direction of NFB procedure.

Good and poor outcome is in almost the same possibilities not only in the present but also previous study (Kotchoubey et al, 1999). About 50\% of the good outcome may be criticized as it is by chance or chance-level. However, it is unlikely because of the following 2 reasons. 1) When we compared good outcome rate between the first one-day sessions and the $2^{\text {nd }}-4^{\text {th }}$ one-day sessions, it was higher in the $2^{\text {nd }}-4^{\text {th }}$ one-day sessions than in the first one-day sessions (30\% vs. $47 \%$ in PD, $36 \%$ vs. $60 \%$ in normals) (although it did not reach the statistical significance). 2) Kotchoubey et al. (1997) showed that many training sessions produced higher rate of good outcome. Namely, after 35 training sessions, 13 patients among 18 patients $(=72 \%)$ showed so-called good training performance. It suggests that longer training is needed to obtain the higher rate of good training outcome.

Since better successful rate in NFB training is essential for clinical applications, more effective training method should be modified. Good regulation of the SCP could provide us with better clinical application. Therefore, it is also very important to delineate any factors to enhance or predict the good training performance for clinical application. For example, since event-related synchronization or desynchronization of background EEG could reflect the intercortical or cortico-subcortical network interaction, a further analysis with SCP may provide us with any predictable information in this regard in the future. Furthermore, future studies 
could clarify the effect of NFB on BP in PD patients with different degree of symptoms.

\section{Conclusions}

Good NFB performance of for negative shifts (negativation) could increase excitatory field potential, probably EPSP of pyramidal cells for early component of BP generation in PD patients and aged normal controls. NFB could enhance the excitability of cortices related to voluntary movement preparation, and a further investigation will be warranted for improvement of clinical motor function of $\mathrm{PD}$ in relation to the $\mathrm{BP}$ restoration. 


\section{References}

Benecke R, Rothwell JC, Dick JP, Day BL, Marsden CD. Disturbance of sequential movements in patients with Parkinson's disease. Brain 1987;110:361-79.

Birbaumer N, Elbert T, Canavan AG, Rockstroh B. Slow potentials of the cerebral cortex and behavior. Physiol Rev 1990;70:1-41.

Cooper R, Osselton JW, Shaw JC. EEG technology, 3rd ed. London: Butterworths, 1980.

Deecke L, Kornhuber HH. An electrical sign of participation of the mesial 'supplementary' motor cortex in human voluntary finger movement. Brain Res 1978;159:473-6.

Dick JPR, Benecke R, Rothwell JC, Day BL, Marsden CD. Simple and complex movements in a patient with infarction of the right supplementary motor area. Mov Disord $1986 ; 1: 255-66$.

Dick JPR, Cantello R, Buruma O, Gioux M, Benecke R, Day BL, et al. The Bereitschaftspotential, L-DOPA and Parkinson's disease. Electroencephalogr Clin Neurophysiol 1987;66:263-74.

Dick JPR, Rothwell JC, Day BL, Cantello R, Buruma O, Gioux M, et al. The Bereitschaftspotential is abnormal in Parkinson's disease. Brain 1989;112:233-44.

Dirnberger G, Duregger C, Trettler E, Lindinger G, Lang W. Fatigue in a simple repetitive motor task: a combined electrophysiological and neuropsychological study. Brain Res 
2004;1028:26-30.

Fumuro T, Matsuhashi M, Mitsueda T, Inouchi M, Matsumoto R, Fujita M, et al.

Neuro-feedback training of slow cortical potentials improves Bereitschaftspotential in normal controls. Jpn J Clin Neurophysiol 2010;38:172-180 (abstract in English).

Hashimoto S, Gemba H, Sasaki K. Analysis of slow cortical potentials preceding self-paced hand movements in the monkey. Exp Neurol 1979;65:218-29.

Hinterberger T, Veit R, Strehl U, Trevorrow T, Erb M, Kotchoubey B, et al. Brain areas activated in fMRI during self-regulation of slow cortical potentials (SCPs). Exp Brain Res 2003;152:113-22.

Hoehn MM, Yahr MD. Parkinsonism: onset, progression and mortality. Neurology $1967 ; 17: 427-42$

Ikeda A, Luders HO, Burgess RC, Shibasaki H. Movement-related potentials recorded from supplementary motor area and primary motor area. Role of supplementary motor area in voluntary movements. Brain 1992;115:1017-43.

Ikeda A, Luders HO, Shibasaki H, Collura TF, Burgess RC, Morris HH,3rd, et al. Movement-related potentials associated with bilateral simultaneous and unilateral movements recorded from human supplementary motor area. Electroencephalogr Clin Neurophysiol 1995;95:323-34. 
Ikeda A, Terada K, Mikuni N, Burgess RC, Comair Y, Taki W, et al. Subdural recording of ictal DC shifts in neocortical seizures in humans. Epilepsia 1996;37:662-74.

Jankovic J. Parkinson's disease: clinical features and diagnosis. J Neurol Neurosurg Psychiatry 2008;79:368-76.

Jurgens U. The efferent and afferent connections of the supplementary motor area. Brain Res 1984;300:63-81.

Kotchoubey B, Blankenhorn V, Froscher W, Strehl U, Birbaumer N. Stability of cortical self-regulation in epilepsy patients. Neuroreport 1997;8:1867-70.

Kotchoubey B, Strehl U, Holzapfel S, Blankenhorn V, Froscher W, Birbaumer N. Negative potential shifts and the prediction of the outcome of neurofeedback therapy in epilepsy. Clin Neurophysiol 1999;110:683-6.

Kotchoubey B, Strehl U, Uhlmann C, Holzapfel S, Konig M, Froscher W, et al. Modification of slow cortical potentials in patients with refractory epilepsy: a controlled outcome study. Epilepsia 2001;42:406-16.

Neshige R, Luders H, Shibasaki H. Recording of movement-related potentials from scalp and cortex in man. Brain 1988;111 ( Pt 3):719-36.

Roland PE, Larsen B, Lassen NA, Skinhoj E. Supplementary motor area and other cortical areas in organization of voluntary movements in man. J Neurophysiol 1980;43:118-36. 
Roland PE, Meyer E, Shibasaki T, Yamamoto YL, Thompson CJ. Regional cerebral blood flow changes in cortex and basal ganglia during voluntary movements in normal human volunteers. J Neurophysiol 1982;48:467-80.

Rossi S, Pasqualetti P, Rossini PM, Feige B, Ulivelli M, Glocker FX, et al. Effects of repetitive transcranial magnetic stimulation on movement-related cortical activity in humans. Cereb Cortex 2000;10:802-8.

Schell GR, Strick PL. The origin of thalamic inputs to the arcuate premotor and supplementary motor areas. J Neurosci 1984;4:539-60.

Schellhorn K, Markert S, Berkes S, Bolten J, Schlegelmilch F. A multifunction EEG-Feedback System. Biomedizinische Technik, 2004;49:766-7.

Shibasaki H, Shima F, Kuroiwa Y. Clinical studies of the movement-related cortical potential (MP) and the relationship between the dentatorubrothalamic pathway and readiness potential (RP). J Neurol 1978;219:15-25.

Shibasaki H, Barrett G, Halliday E, Halliday AM. Cortical potentials following voluntary and passive finger movements. Electroencephalogr Clin Neurophysiol 1980;50:201-13.

Shibasaki H, Hallett M. What is the Bereitschaftspotential? Clin Neurophysiol 2006;117:2341-56.

Tallgren P, Vanhatalo S, Kaila K, Voipio J. Evaluation of commercially available electrodes and 
gels for recording of slow EEG potentials. Clin Neurophysiol 2005;116:799-806.

Tamas LB, Shibasaki H. Cortical potentials associated with movement: a review. J Clin Neurophysiol 1985;2:157-71.

Tomlinson CL, Stowe R, Patel S, Rick C, Gray R, Clarke CE. Systematic review of levodopa dose equivalency reporting in Parkinson's disease. Mov Disord 2010;25:2649-53.

Yazawa S, Ikeda A, Kunieda T, Ohara S, Mima T, Nagamine T, et al. Human presupplementary motor area is active before voluntary movement: subdural recording of Bereitschaftspotential from medial frontal cortex. Exp Brain Res 2000;131:165-77.

Zaidel A, Arkadir D, Israel Z, Bergman H. Akineto-rigid vs. tremor syndromes in Parkinsonism. Curr Opin Neurol 2009;22:387-93. 


\section{Legends}

\section{Fig. 1: Procedural overview}

Subjects performed 3 kinds of tasks in one day in the following order: 1) the first BP recording, followed by 5 min of break, 2) NFB training to regulate SCPs (2-5 times of sub-sessions with 3 min of break), followed by 5 min of break, 3) the second BP recording while subjects made introspective efforts that produced the negative SCP shifts

\section{Fig. 2: Schema of BP components and its measurement}

Baseline: mean amplitude of the movement-related cortical potentials (MRCPs) between 3.0 and $2.6 \mathrm{sec}$ before the trigger onset.

Early BP (E-BP): mean amplitude of the MRCPs between 0.6 and $0.4 \mathrm{sec}$ before the trigger onset.

Pre-movement negative peak (N1): the largest pre-movement negative potentials between -0.5 sec and the trigger onset time measured from the baseline. Late-BP (L-BP): this steeper negativity was obtained by subtracting the e-BP from N1.

\section{Fig. 3: The actual display during NFB trial}


Depending on the task (negative shift or negativation vs. positive shift or positivation), a triangle or reversed triangle appears in the center of the display at the beginning (a, d, a' and d'). During NFB, the actual state of SCPs is shown as a marker of the fish and it moves toward the right $(b, b$ '). When the subjects successfully control SCPs upon the task, a reward-mark appears in the center at the end $(\mathrm{c}, \mathrm{f})$. The same procedures are also done without any appearance of SCP information (without a marker of fish), as shown in d, e, f, d', e' and f'. The details are described in the method (2.3.3).

*Instruction symbol to produce negative SCPs shifts (negativation)

**The shining sun as the reward mark for successful trial

***Instruction symbol to produce positive SCPs shifts (positivation)

**** Since the SCPs shifts fail to exceed the defined target level, no reward mark appears (Cited and modified from Fumuro et al., 2010)

Fig. 4: Example of averaged waveform of the SCPs shift in the good NFB one-day session (left) and poor NFB one-day session (right) (a normal control)

The thick line is the results of negative SCPs performance (negativation), and thin lines for the positive SCPs performance (positivation). $\mathrm{n}=$ the number of trials. *If averaged waveforms of the negativity trials exceed that of the positivity trials at least 3 electrodes 
including $\mathrm{Cz}$ during this time period, it was judged as good performance. Otherwise, judged as poor.

Fig. 5: Effects of NFB performance on $\mathrm{BP}$ for $\mathrm{Cz}$

Each bar indicates the mean difference between the second and first BP amplitudes at

$\mathrm{Cz}$ (the second BP amplitude was subtracted from the first BP amplitude). The second E-BP and

N1 tended to be larger than the first ones in the good NFB one-day sessions, whereas in the poor

NFB performance the second E-BP and N1 tended to be smaller than the first one. L-BP did not show any difference. In good NFB performance, the number of trials of BP was $82 \pm 17$ (mean \pm SD) in the first BP, and $82 \pm 16$ in the second BP. Similarly, in poor NFB performance, the number was $97 \pm 30$ and $92 \pm 23$, respectively.

Fig. 6: Effects of NFB performance on early BP for scalp distribution

Each bar indicates the mean difference between the second and first BP amplitudes at each electrode (the second BP amplitude was subtracted from the first BP amplitude). Cz showed the maximum value with bilateral attenuation.

Fig. 7: Grand-averaged BP in PD patients and normal controls $(\mathrm{Cz})$ by session-based 
analysis

The thick waveform shows the BP measured before the NFB training (first BP). The thin one was the BP (second BP) measured after the training while self-regulated negative SCPs (negativation) was performed. Good NFB performance had the tendency of larger early BP in the second BP than in the first one, whereas the early BP tended to be smaller in the second BP than in the first one in the poor NFB performance in both PD and normal control. $n=$ the number of session.

Fig. 8: Comparison of BP at $\mathrm{Cz}$ between PD patients and normal controls

Each bar indicates the mean BP amplitude at Cz. PD patients had smaller early BP than aged normal controls, but not for late component of BP. 
Fig. 1

First

BP recording
One-day NFB session

NFB training sub-session $\times 2 \sim 5$ (with 3 min of break)

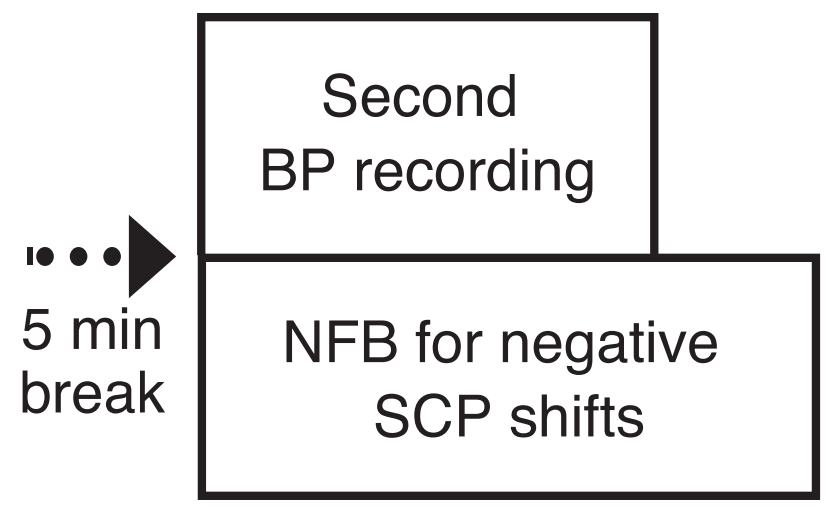


Fig. 2

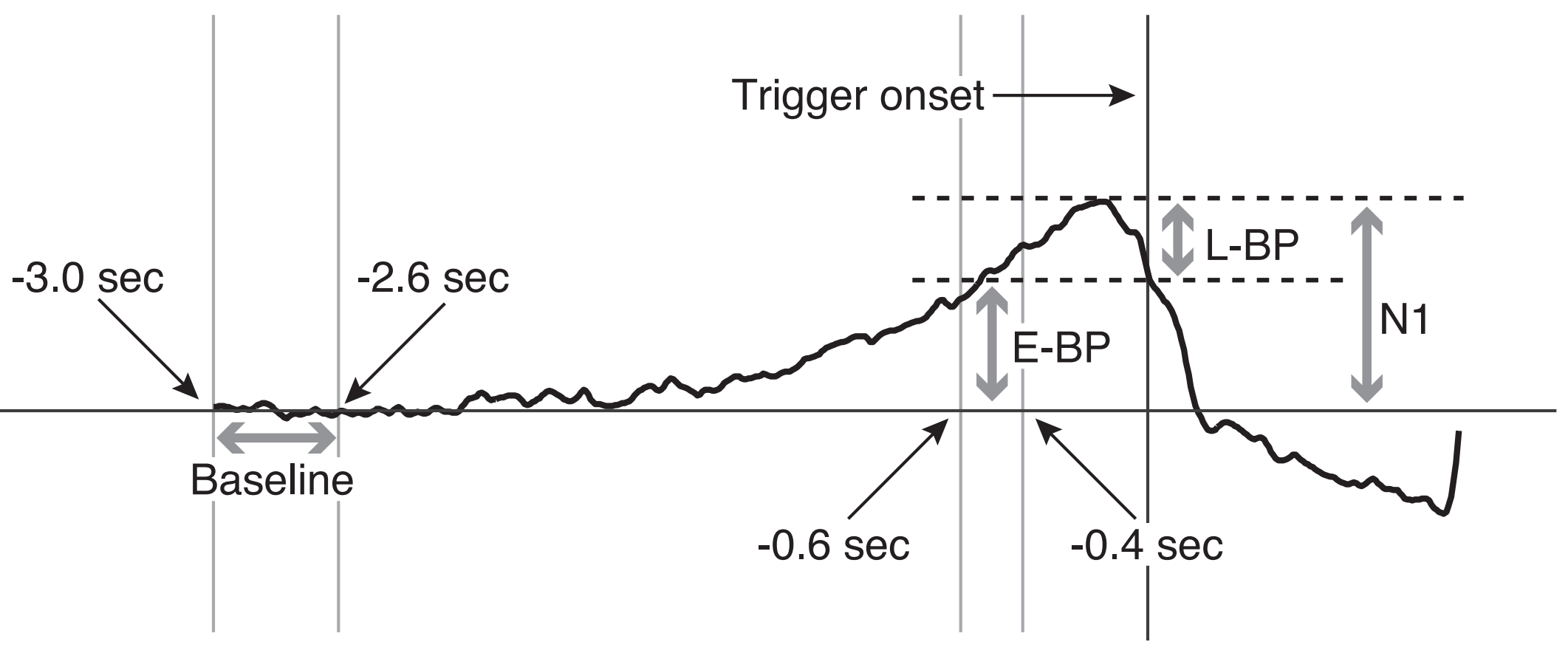




\section{Fig. 3}

(A)

Instruction symbol $=$ negativation, $\mathrm{SCP}$ = negative shifts

Feedback trial

target level baseline

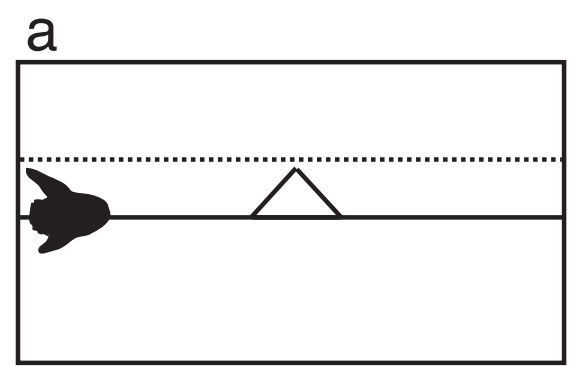

Transfer trial

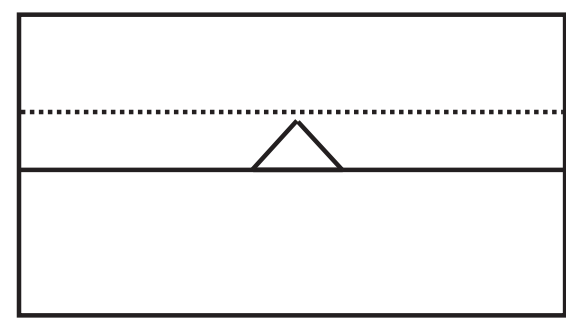

d
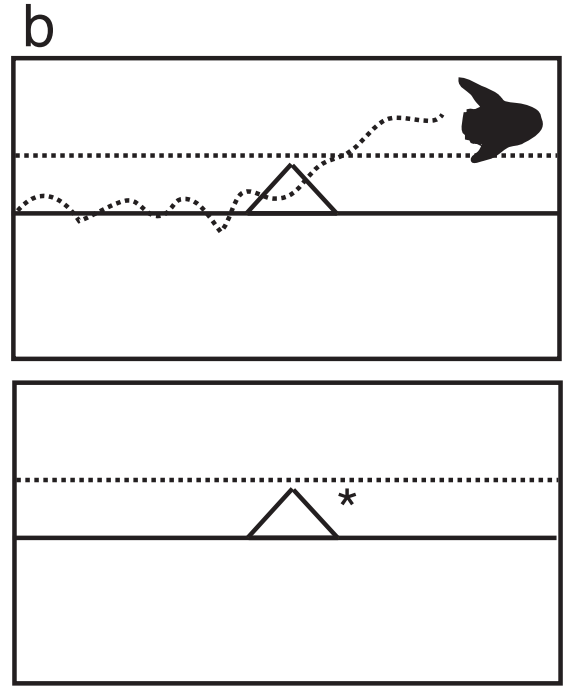

e
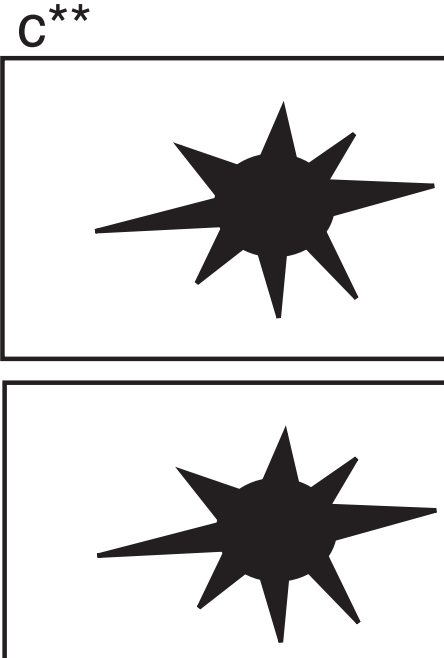

$f^{\star \star}$

(B)

Instruction symbol = positivation, SCPs $=$ negative shifts

Feedback trial

baseline target level

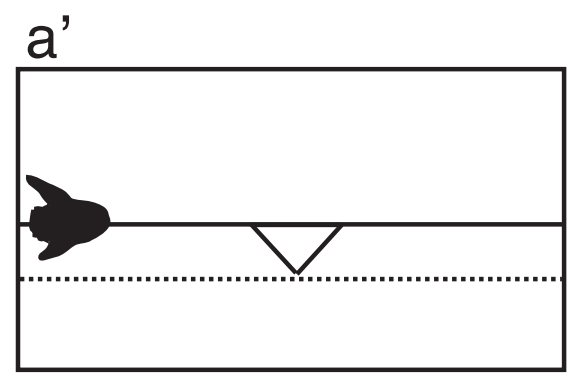

Transfer trial
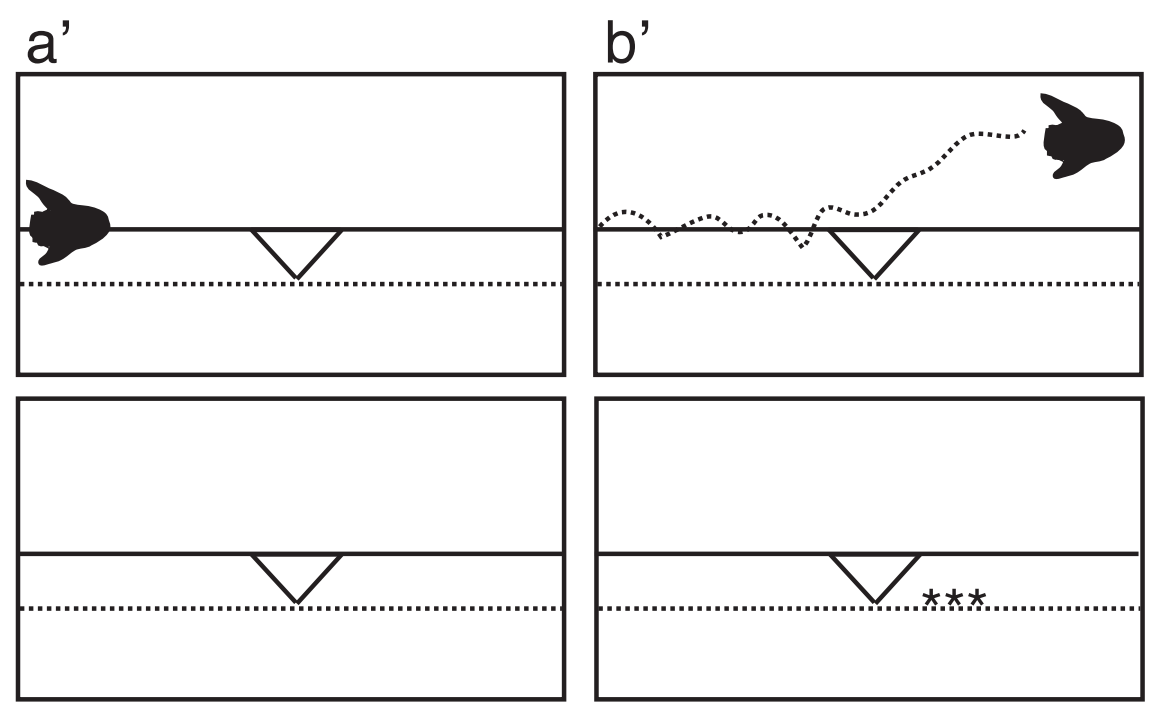

d'

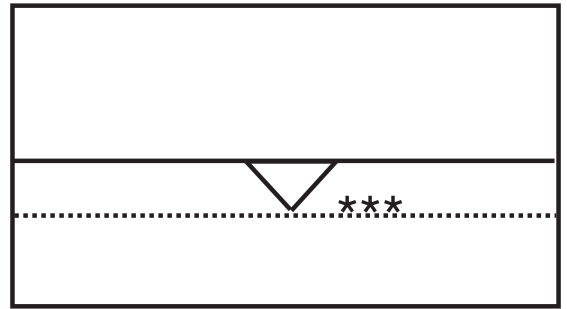

$e^{\prime}$
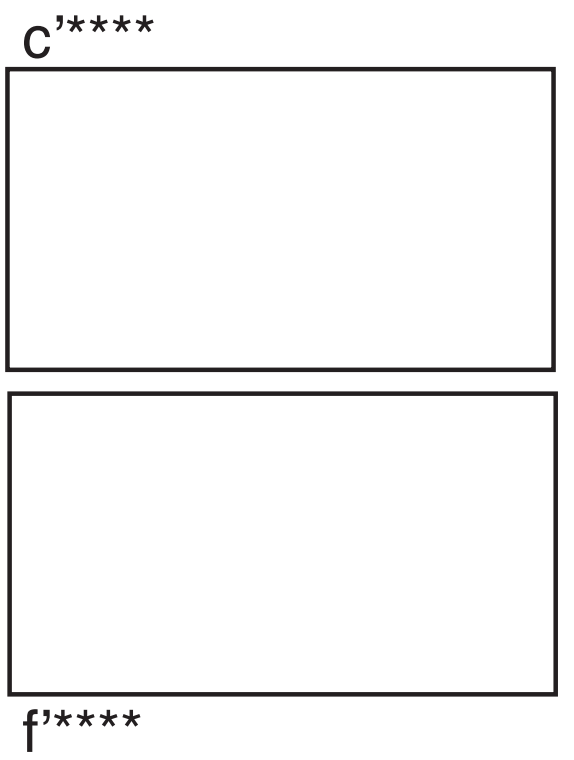
Fig. 4

Good NFB performance

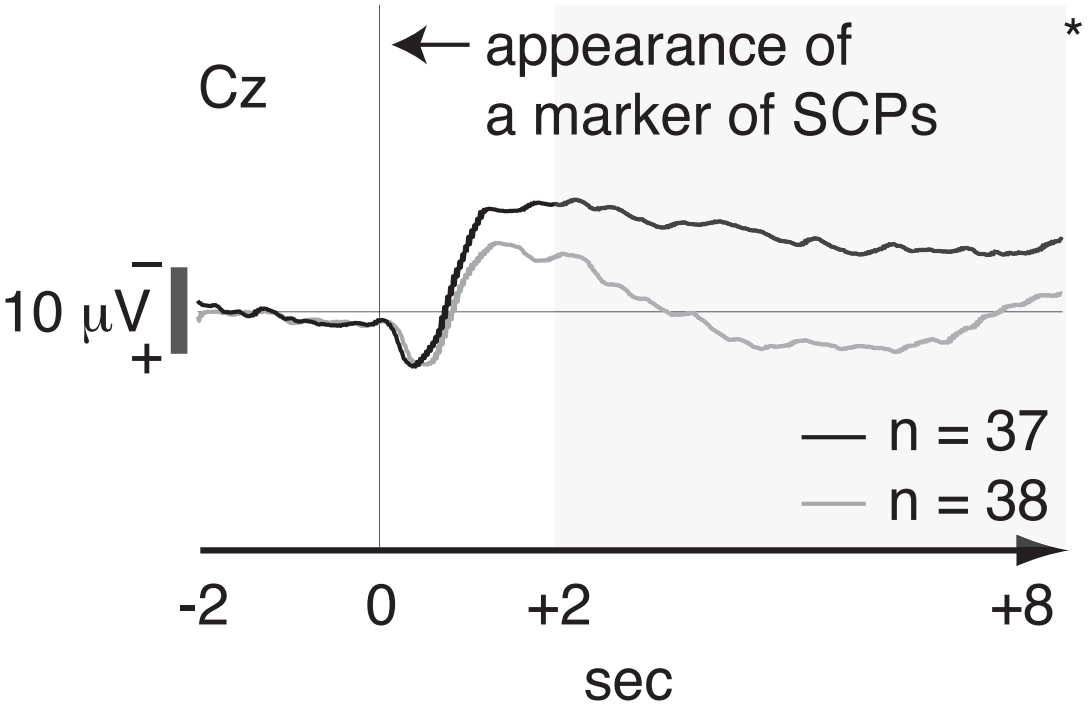

Poor NFB performance

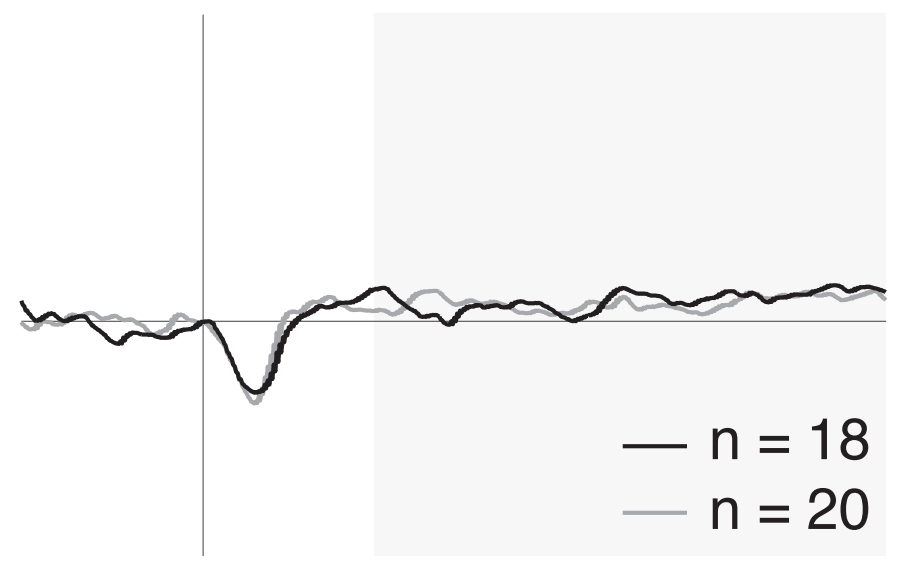

Negative SCPs shifts (negativation) Positive SCPs shifts (positivation) 
Fig. 5

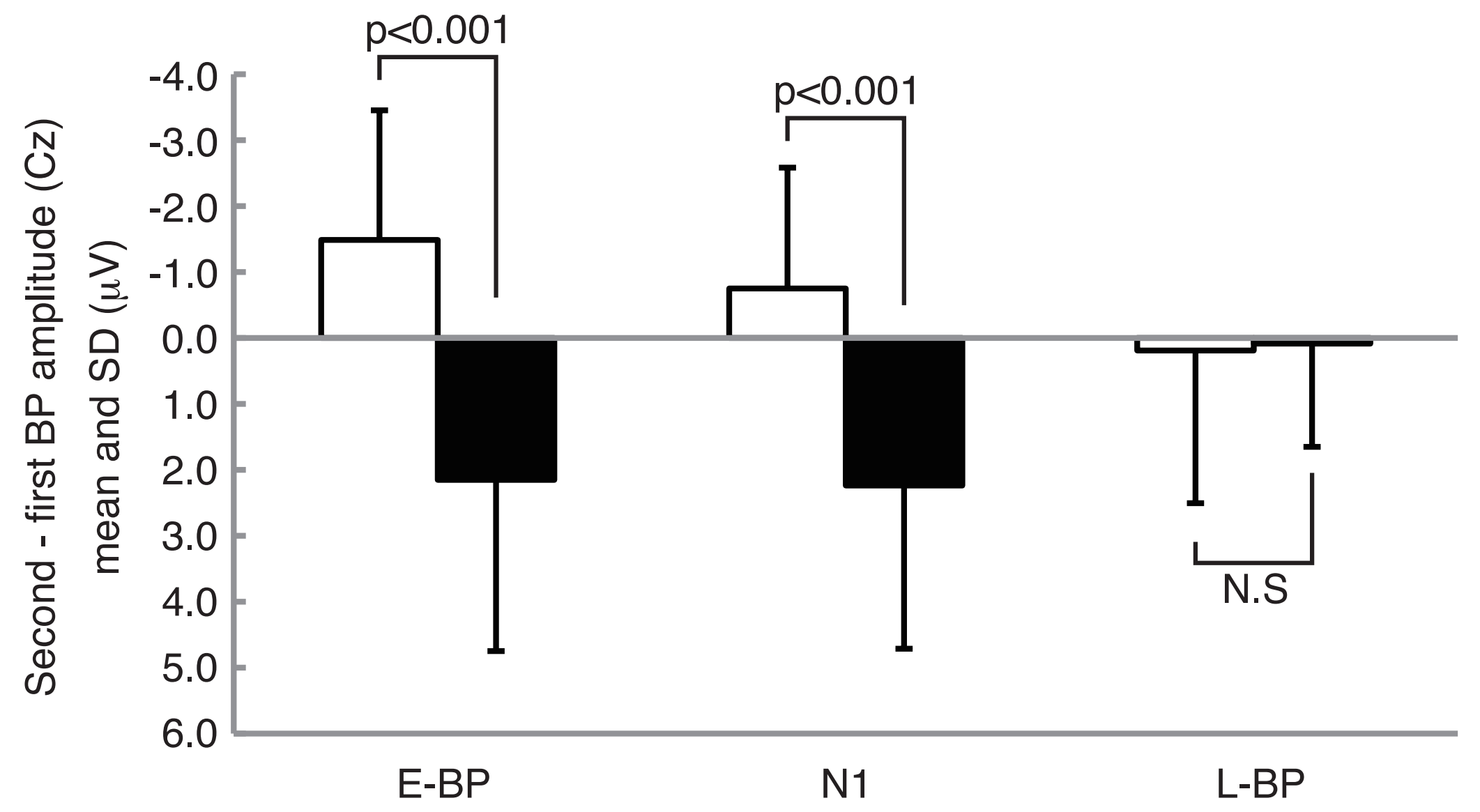

$\square$ Sessions with good NFB performance 
Fig. 7

Sessions with good NFB performance

PD patients
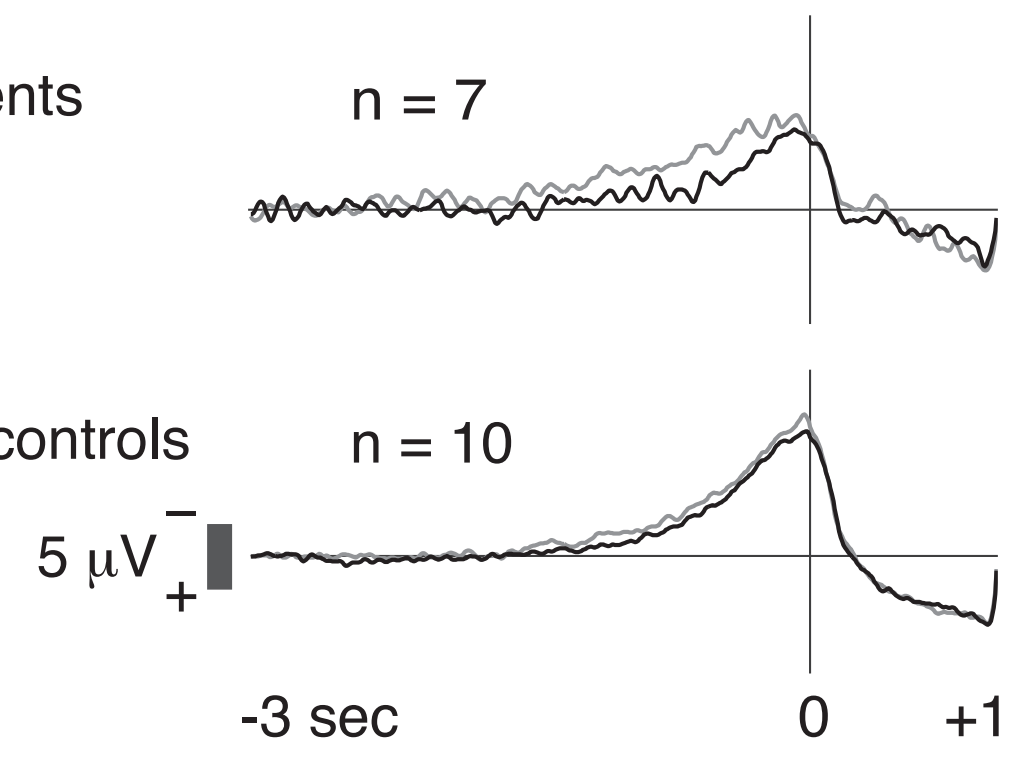

Sessions with poor NFB performance
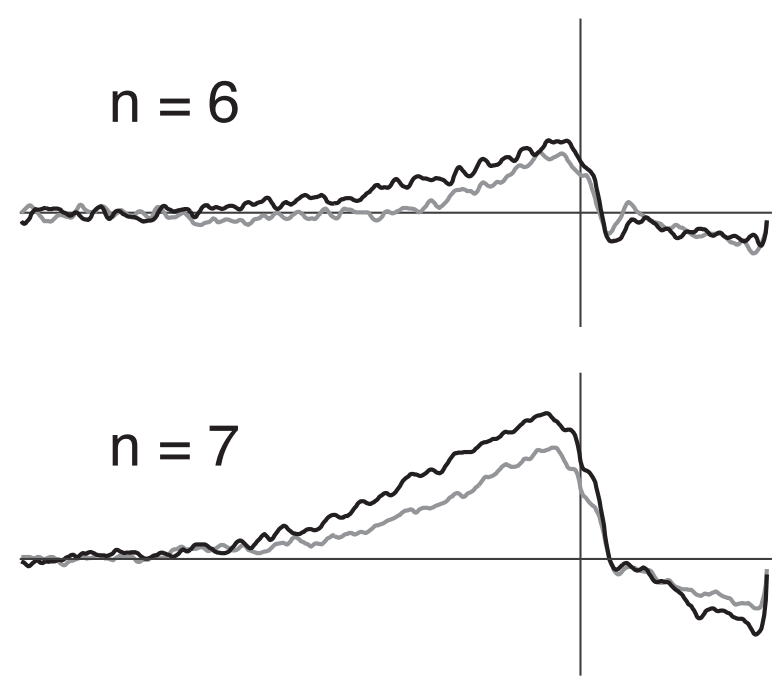

- First BP

- Second BP 
Fig. 8

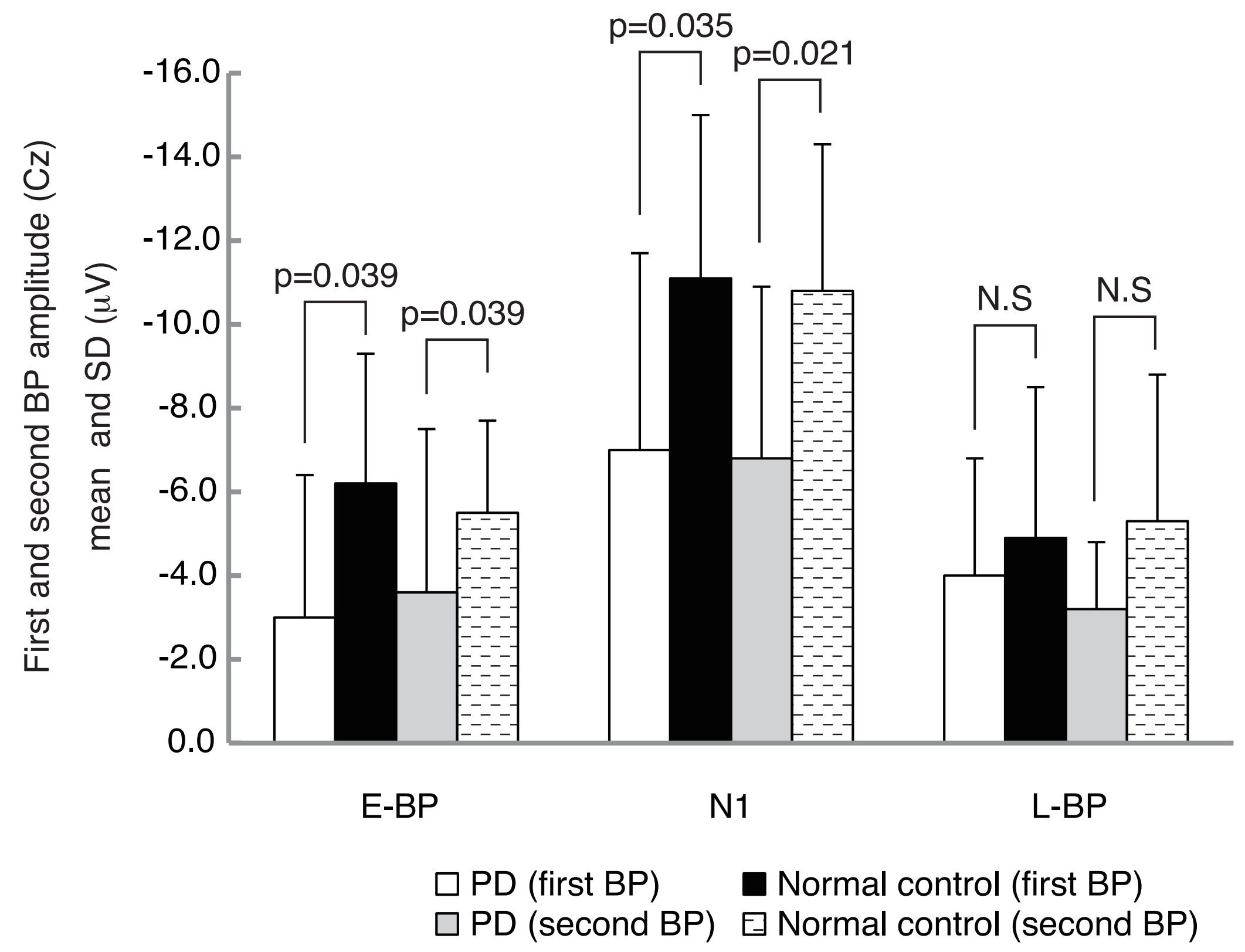




\section{Table 1}

Patient profile

\begin{tabular}{lllll}
\hline $\begin{array}{l}\text { Patient } \\
\text { no. }\end{array}$ & $\begin{array}{l}\text { Age } \\
\text { (years)/gender }\end{array}$ & Diagnosis & $\begin{array}{l}\text { H-Y } \\
\text { grade** }\end{array}$ & $\begin{array}{l}\text { LED** } \\
\text { (mg/d) }\end{array}$ \\
\hline 1 & $71 / \mathrm{W}$ & PD & III & 750 \\
2 & $71 / \mathrm{W}$ & PD & IV & 350 \\
3 & $65 / \mathrm{W}$ & PD & III & 500 \\
4 & $69 / \mathrm{W}$ & PD & III & 600 \\
5 & $68 / \mathrm{W}$ & PD & II-III & 325 \\
6 & $54 / \mathrm{M}$ & PD & I-II & 350 \\
7 & $36 / \mathrm{M}$ & PD & IV & 720 \\
8 & $71 / \mathrm{W}$ & PD & IV & 413 \\
9 & $56 / \mathrm{W}$ & PD & I-II & 125 \\
10 & $71 / \mathrm{W}$ & PD & II & 305 \\
\hline
\end{tabular}

W: woman; M: man; PD: Parkinson's disease

${ }^{*} \mathrm{H}-\mathrm{Y}$ grade $=$ Hoehn and Yahr grade $($ Hoehn and Yahr, 1967)

**LED = levodopa equivalent dose calculated by Tomlinson et al., 2010.

The actual study was done while the medication was one in each patient. 


\section{Table 2}

Analysis of NFB performance and BP (session-based and subject-based)

\begin{tabular}{rll}
\hline & $\begin{array}{l}\text { PD patients } \\
(\mathrm{n}=10)\end{array}$ & $\begin{array}{l}\text { Healthy volunteers } \\
(\mathrm{n}=11)\end{array}$ \\
\hline 1) Session-based analysis & 27 & 31 \\
\hline The total number of one-day sessions & 17 & 20 \\
NFB performance analysis (A) & $8(47 \%)$ & $12(60 \%)$ \\
Good NFB performance & $9(53 \%)$ & $8(40 \%)$ \\
Poor NFB performance & & 17 \\
BP analysis (B) & 13 & 10 \\
Good NFB performance & 7 & 7 \\
Poor NFB performance & 6 & \\
\hline
\end{tabular}

2) Subject-based analysis

Total number of subjects 10 11

The number of subjects for BP analysis (C)

$\begin{array}{ll}7 & 9 \\ 4 & 5 \\ 3 & 4\end{array}$

The number of good NFB group

The number of poor NFB group

4

(A) The first-day sessions ( $\mathrm{n}=10$ and 11) were excluded for further analysis in PD and normals. It was because that in the initial training period subjects could not be familiar with or not good at this internal neuro-feedback process which could distort the finding erroneously.

(B) The first-day sessions ( $\mathrm{n}=10$ and 11) and one-day sessions of poor BP reproducibility $(\mathrm{n}=4$ and 3) were excluded for further analysis in PD and normals.

(C) Subjects having only single one-day session $(\mathrm{n}=1$ and 1$)$ and poor BP reproducibility $(\mathrm{n}=2$ and 1) were excluded from further analysis in PD and normals. 